\title{
HLA DQA1-DQB1-TAP2 haplotypes in IDDM families: no evidence for an additional contribution to disease risk by the TAP2 locus
}

\author{
L. Esposito ${ }^{1}$, V. Lampasona ${ }^{1}$, E. Bosi ${ }^{2}$, F. Poli ${ }^{3}$, M. Ferrari ${ }^{1}$, E. Bonifacio ${ }^{2}$ \\ ${ }^{1}$ Department of Laboratory Medicine, Istituto Scientifico San Raffaele, Milan, Italy \\ ${ }^{2}$ Department of Internal Medicine, Istituto Scientifico San Raffaele, University of Milan, Milan, Italy \\ ${ }^{3}$ Transplantation Immunology and Blood Transfusion Service, Ospedale Maggiore Policlinico, Milan, Italy
}

Summary The TAP2 gene, located in the HLA class II region, encodes a subunit of a transporter involved in the endogenous antigen-processing pathway, and has been suggested to contribute to the genetic risk for insulin-dependent diabetes (IDDM). In order to determine whether the TAP2 locus modulates the risk conferred by HLA DQ loci, HLA DQA1DQB1-TAP2 haplotypes were analysed in 48 IDDM probands, their first degree relatives, and in 62 normal control subjects. A decreased frequency of the TAP2B allele was confirmed in this IDDM cohort (12 vs $28 \%$ in control subjects, $p_{c}<0.05$ ). Analysis of 73 informative meiotic events in IDDM and control families demonstrated a recombination fraction between HLA DQB1 and TAP2 loci of 0.041 (Log of the odds score $=16.5 ; p<10^{-8}$ ) indicating strong linkage between these loci. Family haplotype analysis demonstrated linkage disequilibrium between TAP2 and HLA DQA1-DQB1, and showed that the reduced frequency of TAP2B was associated with its absence on the IDDM susceptible DQA1*0301DQB1*0302 haplotype, its low frequency on
DQA $1 * 0501-\mathrm{DQB} 1 * 0201$, and the association of TAP2B with DQA1*0101-DQB1*0501 haplotypes which were less frequent in IDDM patients. Comparison of transmitted with non-transmitted haplotypes in IDDM families showed a slight but not significant decrease in TAP2B allele frequency on transmitted (3 of 37) vs non-transmitted (2 of 9) HLA DQA1*0501-DQB1*0201 haplotypes. No other differences were observed. Twenty-four unrelated DQA1*0501-DQB1*0201 haplotypes from non-diabetic families had a TAP2B allele frequency $(4 \%)$ similar to that in IDDM haplotypes. These findings suggest that the decreased TAP2B allele frequency in Italian IDDM patients is due to HLA DQ haplotype differences between IDDM patients and control subjects, and do not support a contribution to IDDM risk by the TAP2 locus. [Diabetologia (1995) 38: 968-974]

Key words Antigen presentation, TAP peptide transporter gene, HLA class II, insulin-dependent diabetes mellitus, linkage disequilibrium.
Received: 3 November 1994 and in revised form: 21 February 1995

Corresponding author: Dr. E. Bonifacio, Department of Medicine I, Istituto Scientifico San Raffaele, via Olgettina 60, I20132 Milan, Italy

Abbreviations: ARMS, Amplificatory refractory mutation system; IDDM, insulin-dependent diabetes mellitus; MHC, major histocompatibility complex; PCR, polymerase chain reaction; TAP, transporter associated with antigen processing.
Genes within the major histocompatibility complex (MHC) strongly influence the susceptibility to several autoimmune diseases including insulin-dependent diabetes mellitus (IDDM) [1,2]. Among these, HLA DQ genes provide the major genetic risk for IDDM [3-9]. Together with HLA DR and DP, they encode HLA class II molecules which present antigen peptides to $\mathrm{CD}^{+} \mathrm{T}$ cells [10]. Since HLA DQ alleles conferring susceptibility and/or protection differ at positions critical for peptide binding, IDDM risk conferred by HLA DQ is suggested to act at the level of antigen presentation [11]. 


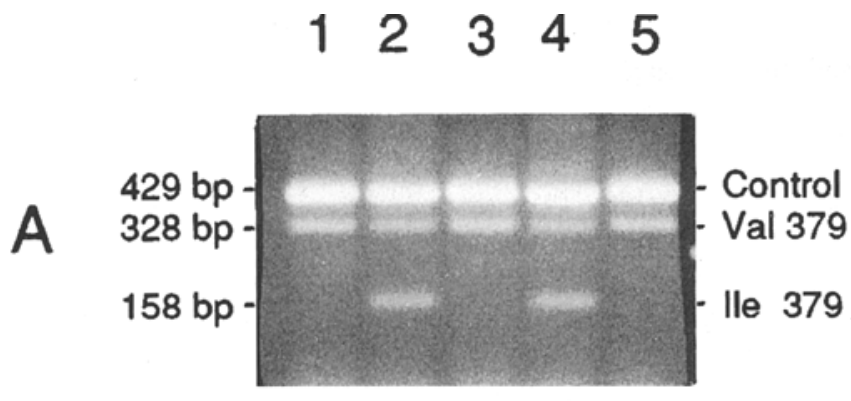

assembly and expression of cytosolic peptides with class I heavy chain and $\beta_{2}$-microglobulin $[21,22]$. Both TAP1 and TAP2 are polymorphic, three TAP1 alleles and six TAP2 alleles having been reported [23]. Allelic frequencies in IDDM patients and normal control subjects show no differences at the TAP1 locus, while in the case of TAP2, an increased frequency of the TAP2A allele and a decreased frequency of the TAP2B allele are found in IDDM patients [24-26]. It remains controversial as to whether these associations are due to linkage disequilibrium between TAP2 and HLA DQ loci [25] or whether this gene confers independent protection or susceptibility for IDDM onset [26]. In order to clarify whether TAP2 modifies the genetic risk for IDDM provided by HLA DQ, we have analysed HLA DQA1DQB1-TAP2 haplotypes in 48 IDDM families, 19 non-diabetic families matched for the IDDM susceptible DQA1*0501-DQB1*0201 haplotype and allele frequencies in 62 normal control subjects. The data indicate that most of the TAP2 association with IDDM can be ascribed to linkage disequilibrium within the HLA class II region.

\section{Subjects and methods}

Fig. 1. (A-C) TAP2 ARMS-PCR analysis of an IDDM family. A Amplified products at position $379 ; \mathbf{B}$ position 565 ; $\mathbf{C}$ position 665. Lane 1 mother (TAP2B/B), lane 2 father (TAP2A/ $\mathrm{D})$, lane 3 unaffected sibling (TAP2A/B), lane 4 proband (TAP2B/TAP2D), lane 5 unaffected sibling (TAP2A/B)

Table 1. TAP2 allele frequencies in IDDM probands and control subjects

\begin{tabular}{lll}
\hline TAP2 allele & $\begin{array}{l}\text { IDDM probands } \\
n=48\end{array}$ & $\begin{array}{l}\text { Control subjects } \\
n=62\end{array}$ \\
\hline TAP2A & $70(73 \%)$ & $75(60 \%)$ \\
TAP2B & $12(12 \%)$ & $35(28 \%)^{\mathrm{a}}$ \\
TAP2C & $11(11 \%)$ & $11(9 \%)$ \\
TAP2D & $2(2 \%)$ & $0(0 \%)$ \\
TAP2E & $1(1 \%)$ & $2(2 \%)$ \\
TAP2F & $0(0 \%)$ & $1(1 \%)$ \\
\hline
\end{tabular}

${ }^{a} p_{c}<0.05 ; p$ values were corrected for the number of TAP2 alleles $(n=6)$

Several other genes within the MHC are involved in antigen presentation and may be relevant to the genetics of IDDM [12]. These include a cluster of genes located between HLA DQ and HLA DP which are necessary for successful cell surface expression and presentation of peptides with HLA class I and class II molecules [13-18]. Of these, the TAP1 and TAP2 loci encode subunits of an ATP-dependent transporter which delivers endogenous peptides from the proteosome complex to the HLA class I molecule $[19,20]$. This function is essential for stable

Subjects. Forty-eight unrelated IDDM probands and their firstdegree relatives were randomly selected from the San Raffaele Hospital IDDM family study in the Lombardy region of northern Italy. Diagnosis of IDDM in probands was made according to the World Health Organization criteria. Twenty-three probands were male, 25 were female and their average age at IDDM onset was 16.6 years (range $4.3-31.6$ years). Each family had at least one unaffected sibling, who was less than age 32 years. Sixty-two unrelated non-diabetic individuals from the same geographical area with a mean age of 57.5 years were also recruited for the investigation. They were randomly selected to represent the general population of Milan, did not have IDDM or non-insulin-dependent diabetes, or a family history of the disorders, and all had a normal daily glucose profile. In order to compare HLA DQA1-DQB1-TAP2 haplotypes with those from HLA DQ matched non-diabetic families, DNA was provided by the North Italian Transplant centre from 19 non-diabetic families which contained at least one DQA1*0501-DQB1*0201 haplotype.

$D Q A 1-D Q B 1$ typing. Genomic DNA was extracted from peripheral blood lymphocytes according to standard techniques [27]. The second exon of the DQA1 and DQB1 genes was amplified by polymerase chain reaction (PCR) and typed with allele specific oligonucleotide probes specific for DQB1 codons 54-59 and DQA1 codons 52,72-74 according to the 11th HLA international workshop protocols.

TAP2 ARMS PCR analysis. TAP2 ARMS (amplificatory refractory mutation system) PCR analysis was performed as previously described [23]. Three polymorphic sites were independently typed: position 379 (A for $\mathrm{G}, \mathrm{Ile}_{379}$ for $\mathrm{Val}_{379}$ ) position 565 ( $\mathrm{A}$ for $\mathrm{G}, \mathrm{Thr}_{565}$ for $\mathrm{Ala}_{565}$ ), position 665 ( $\mathrm{G}$ for $\mathrm{A}, \mathrm{Ala}_{665}$ for $\left(\mathrm{Th}_{665}\right)$. Four primers were used for each site: two specific for one of the two variants, the others complementary to the flanking regions as control. PCR reactions were performed in 
Table 2. HLA DQA1-DQB1-TAP2 haplotypes in parents of IDDM families

\begin{tabular}{|c|c|c|c|c|c|}
\hline & TAP2A & TAP2B & TAP2C & Others & Total \\
\hline DQA1*0201-DQB1*0201 & 9 & 3 & 0 & 0 & 12 \\
\hline DQA1*0501-DQB1*0201 & $38(0.41)^{\mathrm{a}}$ & 5 & 1 & 2 & 46 \\
\hline DQA1*0501-DQB1*0301 & 15 & 3 & $12(0.76)^{\mathrm{a}}$ & 0 & 30 \\
\hline DQA1*0301-DQB1*0302 & $23(0.86)^{\mathrm{a}}$ & $0(1.0)^{\mathrm{a}}$ & 0 & 1 & 24 \\
\hline DQA1*0101-DQB1*0501 & 11 & $10(0.71)^{\mathrm{a}}$ & 0 & 1 & 22 \\
\hline DQA1*0102-DQB1*0502 & 8 & 3 & 2 & 0 & 13 \\
\hline DQA1*0102-DQB1*0602 & 9 & 1 & 0 & 1 & 11 \\
\hline Others & 10 & 1 & 4 & 1 & 15 \\
\hline Total & 123 & 26 & 19 & 6 & 174 \\
\hline
\end{tabular}

${ }^{a}$ Standardized linkage disequilibrium $\mathrm{D}^{\prime}$ is in parentheses

a $50 \mu \mathrm{l}$ final volume containing $500 \mathrm{ng}$ of genomic DNA, $0.25 \mu \mathrm{mol} / 1$ of each oligonucleotide primer, $200 \mu \mathrm{mol} / 1 \mathrm{dNTPs}$, $50 \mathrm{mmol} / 1 \mathrm{KCl}, 10 \mathrm{mmol} / 1 \mathrm{MgCl}_{2}, 0.1 \%$ gelatine, 1.2 units of Taq DNA polymerase (Perkin-Elmer Cetus, Norwalk, Conn., USA). Amplifications were carried out in a DNA Thermal Cycler (TC 9600 , Perkin-Elmer Cetus) for 30 cycles of denaturation $\left(95^{\circ} \mathrm{C}\right.$ for $\left.30 \mathrm{~s}\right)$, annealing $\left(58^{\circ} \mathrm{C}\right.$ or $60^{\circ} \mathrm{C}$ for $\left.1 \mathrm{~min}\right)$ and extension $\left(72^{\circ} \mathrm{C}\right.$ for $1 \mathrm{~min}$ ) followed by a final 5 -min extension step. Amplified products were visualised on a $2 \%$ agarose gel stained with ethidium bromide. Amplification at each site resulted in a constant control band and minor bands of specific sizes corresponding to the allelic variants (Fig. 1). The alleles TAP2A-E were assigned as described by Powis et al. [23]; TAP2F was assigned to the variant $\mathrm{Ile}_{379}-\mathrm{Ala}_{565}$ - Ala $_{665}$.

\section{Statistical analysis}

Gene frequencies were evaluated by counting individual alleles and dividing by the total number of TAP2 alleles. Statistically significant differences between the IDDM probands and unrelated non-diabetic control subjects were assessed using the chi-square test; $p$ values were corrected for multiple comparisons.

HLA DQA1-DQB1-TAP2 haplotypes were assigned after evaluating the genotype data for all family members. Haplotypes could not be assigned in three families due to recombination, and a further six parental haplotypes could not be unambiguously determined. Haplotypes occurring in probands were defined as transmitted, while those occurring only among unaffected family members were defined as non-transmitted. It was possible to assign 90 transmitted haplotypes and 84 non-transmitted haplotypes. Differences between the frequency of transmitted and non-transmitted haplotypes were assessed using the chi-square test according to Thomson [28]. An additional comparison of the TAP2 distribution within DQA1*0501-DQB1*0201 haplotypes from IDDM patients was made with 24 unrelated DQA1*0501-DQB1*0201 haplotypes from 19 families with no immediate history of IDDM.

The extent of genetic linkage between DQB1 and TAP2 loci was determined by calculating the Logarithm of the odds (Lod) score of the recombination fraction in all meioses which occurred in parents heterozygous at both loci, and included IDDM and control families [29]. The degree of linkage disequilibrium between HLA DQ haplotypes and TAP2 was obtained by calculating $D^{\prime}$ (delta/delta max ) [30] on the frequency of haplotypes and alleles in the parents of IDDM families [31]. All $p$ values were corrected for the number of potential comparisons $(n=32)$.

\section{Results}

TAP2 allele frequencies. The TAP2B allele frequency was decreased in IDDM patients $(12 \%)$ vs control subjects $(28 \%),\left(p_{\mathrm{c}}<0.05\right)$ and the TAP2A frequency was slightly but not significantly increased (73 vs $60 \%$ ) (Table 1). Overall, TAP2A was present in $92 \%$ of patients and $84 \%$ of control subjects, and TAP2B in $23 \%$ of patients and $48 \%$ of control subjects $\left(p_{\mathrm{c}}=0.06\right)$.

Recombination analysis. Among the IDDM and control families there were 30 families in which at least one parent was heterozygous at both DQB1 and TAP2 loci, and within these families 73 meiotic events informative for assessment of recombination between these loci. Three DQB1-TAP2 recombinations were observed giving a recombination fraction of 0.041 and an Lod score of $16.5\left(p<10^{-8}\right)$.

Linkage disequilibrium. Association of HLA DQ haplotypes and TAP2 alleles in parents of IDDM families is shown in Table 2. The IDDM susceptible DQA1*0301-DQB1*0302 haplotype was almost always found with the TAP2A allele and never with TAP2B. TAP2A was on 23 of $24(96 \%)$ of DQA1*0301-DQB1*0302 haplotypes compared to 100 of $150(67 \%)$ of non-DQA1*0301-DQB1*0302 haplotypes $\left(\chi^{2}=7.1, p_{\mathrm{c}}=0.22\right)$. The TAP2B allele was most frequent on the DQA $1 * 0101-\mathrm{DQB} 1 * 0501$ haplotype, present on 10 of 22 (45\%) of these haplotypes compared to 16 of $152(9.5 \%)$ nonDQA1*0101-DQB $1 * 0501$ haplotypes $\left(\chi^{2}=15.8\right.$, $\left.p_{\mathrm{c}}<0.005\right)$. TAP2C was most frequent on the DQA1*0501-DQB1*0301 haplotype, present on 12 of $30(40 \%)$ of these haplotypes compared to 7 of 138 (5\%) non-DQA1*0501-DQB1*0301 haplotypes $\left(\chi^{2}=26.6, p_{\mathrm{c}}<0.00005\right)$.

Transmitted vs non-transmitted haplotypes. The frequency of the DQA1*0501-DQB1*0201-TAP2A haplotype was increased $\left(p_{\mathrm{c}}<0.005\right)$ and the DQA1*0501-DQB1*0301-TAP2A haplotype reduced $\left(p_{\mathrm{c}}<0.05\right)$ in transmitted haplotypes (Ta- 
ble 3). The DQA1*0301-DQB1*0302-TAP2A haplotype was slightly but not significantly increased in transmitted haplotypes. DQA1*0102-DQB1*0602TAP2A and DQA $1 * 0101-D Q B 1 * 0501-T A P 2 B$ haplotypes were slightly but not significantly reduced in transmitted haplotypes.

Amongst the DQA1*0501-DQB1*0201 haplotypes, TAP2B allele was present on $3(8 \%)$ of 37 transmitted compared to $2(22 \%)$ of 9 non-transmitted haplotypes $\left(p_{\text {uncorrected }}=0.24\right)$. No other differ-

Table 3. Transmitted and non-transmitted HLA DQA1DQB1-TAP2 haplotypes in IDDM families

\begin{tabular}{|c|c|c|}
\hline Haplotypes & $\begin{array}{l}\text { Trans- } \\
\text { mitted } \\
n=90\end{array}$ & $\begin{array}{l}\text { Non-trans- } \\
\text { mitted } \\
n=84\end{array}$ \\
\hline $\begin{array}{r}\mathrm{DQA} 1 * 0201-\mathrm{DQB} 1 * 0201-\mathrm{TAP} 2 \mathrm{~A} \\
\text {-TAP2B }\end{array}$ & $\begin{array}{l}4(4 \%) \\
2(2 \%)\end{array}$ & $\begin{array}{l}5(6 \%) \\
1(1 \%)\end{array}$ \\
\hline $\begin{array}{r}\text { DQA } 1 * 0301-\mathrm{DQB} 1 * 0201-\mathrm{TAP} 2 \mathrm{~A} \\
-\mathrm{TAP} 2 \mathrm{C}\end{array}$ & $\begin{array}{l}3(3 \%) \\
0(0 \%)\end{array}$ & $\begin{array}{l}0(0 \%) \\
1(1 \%)\end{array}$ \\
\hline $\begin{array}{r}\text { DQA1*0501-DQB1*0201-TAP2A } \\
\text {-TAP2B } \\
\text {-TAP2C } \\
\text {-TAP2D }\end{array}$ & $\begin{array}{l}31(34 \%) \\
3(3 \%) \\
1(1 \%) \\
2(2 \%)\end{array}$ & $\begin{array}{l}7(8 \%)^{\mathrm{a}} \\
2(2 \%) \\
0(0 \%) \\
0(0 \%)\end{array}$ \\
\hline $\begin{array}{r}\text { DQA1*0301-DQB1*0301-TAP2A } \\
\text {-TAP2C } \\
\text {-TAP2D }\end{array}$ & $\begin{array}{l}1(1 \%) \\
1(1 \%) \\
0(0 \%)\end{array}$ & $\begin{array}{l}1(1 \%) \\
0(0 \%) \\
1(1 \%)\end{array}$ \\
\hline $\begin{array}{r}\text { DQA } 1 * 0501-\mathrm{DQB} 1 * 0301-\mathrm{TAP} 2 \mathrm{~A} \\
-\mathrm{TAP} 2 \mathrm{~B} \\
\text {-TAP2C }\end{array}$ & $\begin{array}{l}1(1 \%) \\
0(0 \%) \\
4(4 \%)\end{array}$ & $\begin{array}{l}14(17 \%)^{\mathrm{b}} \\
3(3 \%) \\
8(10 \%)\end{array}$ \\
\hline $\begin{array}{r}\text { DQA } 1 * 0301-\mathrm{DQB} 1 * 0302-\mathrm{TAP} 2 \mathrm{~A} \\
\text {-TAP2E }\end{array}$ & $\begin{array}{c}16(18 \%) \\
1(1 \%)\end{array}$ & $\begin{array}{l}7(8 \%) \\
0(0 \%)\end{array}$ \\
\hline $\begin{array}{r}\text { DQA } 1 * 0301-D Q B 1 * 0303-T A P 2 A \\
\text {-TAP2B }\end{array}$ & $\begin{array}{l}1(1 \%) \\
1(1 \%)\end{array}$ & $\begin{array}{l}0(0 \%) \\
0(0 \%)\end{array}$ \\
\hline DQA1*0401-DQB1*0402-TAP2A & $1(1 \%)$ & $1(1 \%)$ \\
\hline $\begin{array}{r}\text { DQA1*0101-DQB1*0501-TAP2A } \\
\text {-TAP2B } \\
\text {-TAP2F }\end{array}$ & $\begin{array}{l}4(4 \%) \\
2(2 \%) \\
0(0 \%)\end{array}$ & $\begin{array}{l}7(8 \%) \\
8(10 \%) \\
1(1 \%)\end{array}$ \\
\hline $\begin{array}{r}\text { DQA } 1 * 0102-\mathrm{DQB} 1 * 0502-\mathrm{TAP} 2 \mathrm{~A} \\
-\mathrm{TAP} 2 \mathrm{~B} \\
\text {-TAP2C }\end{array}$ & $\begin{array}{l}5(6 \%) \\
2(2 \%) \\
2(2 \%)\end{array}$ & $\begin{array}{l}3(3 \%) \\
1(1 \%) \\
0(0 \%)\end{array}$ \\
\hline $\begin{array}{r}\text { DQA1*0103-DQB1*0503-TAP2A } \\
\text {-TAP2C }\end{array}$ & $\begin{array}{l}0(0 \%) \\
1(1 \%)\end{array}$ & $\begin{array}{l}1(1 \%) \\
1(1 \%)\end{array}$ \\
\hline $\begin{array}{r}\text { DQA } 1 * 0102-\mathrm{DQB} 1 * 0602-\mathrm{TAP} 2 \mathrm{~A} \\
\text {-TAP2B } \\
\text {-TAP2E }\end{array}$ & $\begin{array}{l}1(1 \%) \\
0(0 \%) \\
0(0 \%)\end{array}$ & $\begin{array}{l}8(10 \%) \\
1(1 \%) \\
1(1 \%)\end{array}$ \\
\hline DQA1*0101-DQB1*0601-TAP2A & $0(0 \%)$ & $1(1 \%)$ \\
\hline
\end{tabular}

${ }^{\mathrm{a}} p_{\mathrm{c}}<0.005 ;{ }^{\mathrm{b}} p_{\mathrm{c}}<0.05$

$p$ values were corrected for the number of haplotypes $(n=31)$ ences in TAP2 allele distribution between HLA DQ matched transmitted and non-transmitted haplotypes were found. To further evaluate whether TAP2B might confer additional protection on the DQA1*0501-DQB1*0201 haplotype, control families were examined. TAP2B was on $1(4 \%)$ of 24 unrelated DQA1*0501-DQB1*0201 haplotypes from families without IDDM. This was similar to its frequency in HLA DQA1*0501-DQB1*0201 haplotypes found in IDDM patients (Table 4).

\section{Discussion}

Our results confirmed the altered distribution of TAP2 alleles in IDDM patients compared to control subjects. The strongest TAP2 allele association with IDDM was a decreased frequency of TAP2B. A previous case-control study in France [26] suggests that this allele provides dominant protection against developing IDDM. A separate study in IDDM patients in Scandinavia [25] reports that its reduced frequency is due to linkage disequilibrium between TAP2 and HLA DQ. More recently, two additional case-control studies have also reported conflicting results $[32,33]$. A study in Spanish IDDM patients and control subjects shows IDDM association with TAP2 $* 01$ alleles which include TAP2A, C, D and E, but not TAP2B and F, and suggests this is not due to linkage disequilibrium [32]. A study in the Sardinian population, however, reports only minor differences in TAP2 allele distributions between patients and control subjects which can be attributed to linkage disequilibrium between HLA DR, DQ, and TAP2, and concludes that a primary association between IDDM and TAP2 is unlikely [33]. We have analysed northern Italian IDDM families to identify HLA DQA1-DQB1-TAP2 haplotypes. Family analysis should improve the power to determine whether the TAP2 allele associations with IDDM are primary or secondary to HLA DQ.

Genotyping in our IDDM families demonstrated an association of TAP2 alleles with particular HLA DQ haplotypes. TAP2A was preferentially found on DQA1*0301-DQB1*0302 haplotypes, TAP2B on DQA1*0101-DQB1*0501 haplotypes, and TAP2C on DQA1*0501-DQB1*0301 haplotypes. TAP2B was absent on all DQA1*0301-DQB1*0302 haplotypes and found in only $8 \%$ of DQA1*0501-

Table 4. TAP2 allele distribution on HLA DQA1*0501-DQB1*0201 haplotypes in IDDM and control families

\begin{tabular}{lccc}
\hline Haplotype & $\begin{array}{l}\text { Transmitted (IDDM) } \\
(n=37)\end{array}$ & $\begin{array}{l}\text { Non-transmitted } \\
(n=9)\end{array}$ & $\begin{array}{l}\text { Control families } \\
(n=24)\end{array}$ \\
\hline DQA1*0501-DQB1*0201-TAP2A & $31(84 \%)$ & $7(78 \%)$ & $20(83 \%)$ \\
DQA1*0501-DQB1*0201-TAP2B & $3(8 \%)$ & $2(22 \%)$ & $1(4 \%)$ \\
DQA1*0501-DQB1*0201-TAP2C & $1(3 \%)$ & $0(0 \%)$ & $1(4 \%)$ \\
DQA1*0501-DQB1*0201-TAP2D & $2(5 \%)$ & $0(0 \%)$ & $2(8 \%)$ \\
\hline
\end{tabular}


DQB1*0201 haplotypes. These two HLA DQ haplotypes accounted for $59 \%$ of haplotypes in the IDDM patients. Furthermore, TAP2B was in linkage disequilibrium with, and most frequently found on, the DQA1*0101-DQB1*0501 haplotype which has a reduced frequency in IDDM patients. Therefore, as in the Scandinavian study [25], the decreased TAP2B allele frequency in our IDDM cohort could be accounted for by increased frequencies of DQA1*0301-DQB1*0302 and DQA1*0501DQB $1 * 0201$ and, in particular, a reduced frequency of DQA1*0101-DQB $1 * 0501$.

This study shows clear linkage disequilibrium between TAP2 and HLA DQ. Similar associations between DQA1*0101-DQB1*0501 or DRB1*01 and TAP2B are shown in previous studies $[25,26,32,33]$, while linkage disequilibrium between TAP2C and DRB1*11 which is found on DQA1*0501DQB $1 * 0301$ haplotypes is also present in the Sardinian cohorts [33], and either not tested [25, 32] or not shown [26] in other studies. Linkage disequilibrium between the more common IDDM-susceptible HLA DRB1*0301-DQA1*0501-DQB1*0201 and DRB1*04-DQA1*0301-DQB1*0302 haplotypes is, however, controversial. Rønningen et al. [25] show statistically significant delta values, albeit only when $p$ values are uncorrected, between each of these haplotypes and TAP2A in Norwegian random-control subjects. None of the other studies, including our own (data not shown), demonstrate statistically significant associations of TAP2A with these haplotypes in random control subjects. The absence of statistically significant linkage disequilibria between DRB1*04-DQA $1 * 0301-D Q B 1 * 0302$ or DRB1*0301-DQA1*0501-DQB1*0201 and TAP2A in random control subjects may, however, be due to insufficient statistical power (large type II error) arising from the infrequency of alleles other than TAP2A in each population. In these cases it is inappropriate to exclude linkage disequilibrium on the basis of a non-significant delta value. Cucca et al. [33] found a significantly higher frequency of TAP2A in DRB1*0301-DQA1*0501-DQB1*0201 homozygous IDDM patients and control subjects compared to random control subjects. This is also the case in the Scandinavian study, and in support of this, we found that DQA1*0501-DQB1*0201 haplotypes have a higher frequency of TAP2A than do the remaining haplotypes $(p=0.06$, Table 2$)$. Furthermore, in the Scandinavian, Spanish and our northern Italian cohorts, DQB $1 * 0302$ was almost never found in the absence of TAP2A, and they are, in fact, clearly associated. We suggest, therefore, that each of the studies, which are on cohorts distinct in their geography, HLA class I and II distributions, and IDDM incidence [34], are consistent in their findings, and support the existence of linkage disequilibrium between the HLA DQ and TAP2.
Linkage disequilibrium of alleles at several loci within the HLA class II region, complicates analyses of contribution to disease risk by separate loci. The region of linkage disequilibrium around HLA DQ is reported to include TAP2, but not the more centromeric TAP1 and DP loci [35]. In the case of the TAP2 locus, allele associations with IDDM appear to be secondary to the disease association with HLA DQ. This implies that TAP2 allele typing is unlikely to improve assessment of risk for IDDM over that provided by DQ typing alone. However, it does not exclude a role for the allelic differences in the pathogenesis of IDDM. One approach which may help clarify whether a linked locus modulates risk is to examine haplotype transmission to probands. A reduced transmission of particular HLA DQ haplotypes when they carry TAP2B would support protection by this allele. DQA1*0301-DQB1*0302 was uninformative for this purpose since it was never found with TAP2B in either transmitted or non-transmitted haplotypes. TAP2B was slightly less frequent on DQA1*0501-DQB1*0201 transmitted haplotypes, but this decreased frequency in transmitted vs nontransmitted groups was not statistically significant. Furthermore, comparison of transmitted vs nontransmitted haplotypes is conditional on either the absence of recombination or linkage disequilibrium [36]. The frequencies of TAP2B alleles on DQA1*0501-DQB $1 * 0201$ transmitted haplotypes were therefore compared to those in matched control families. There were no differences between these groups. This is consistent with the findings of Rønningen et al. [25] and Cucca et al. [33] who found no differences between TAP2 allele frequencies in IDDM patients and control subjects homozygous for this haplotype.

These studies stress the importance of using HLAmatched control subjects in analyses of IDDM susceptibility [37]. In neither study in which an independent contribution to IDDM risk was attributed to the TAP2 locus were the TAP2 allele frequencies in IDDM patients compared to HLA-matched or HLA-DQ homozygous control subjects $[25,32]$. In contrast, where such comparisons were made, differences in TAP2 allele frequencies between IDDM patients and control subjects were found to be secondary to HLA-DQ associations with IDDM. We conclude that HLA DQA1-DQB1-TAP2 haplotype analysis within Italian families also suggests that the decreased TAP2B allele frequency in IDDM patients is secondary to HLA DQ haplotype associations with disease. We do not exclude a role for TAP2 allelic differences in the pathogenesis of IDDM, but suggest that any contribution to IDDM risk will be small. TAP2 allele typing is, therefore, unlikely to be useful for IDDM risk assessment. The demonstration of additional risk to that attributable to HLA DQ will require analysis of very large cohorts of IDDM families 
or alternative approaches [38]. This is also likely to be the case for other loci within the MHC which are in linkage disequilibrium with HLA DQ.

Acknowledgements. We wish to thank Drs. M. R. Pastore and A. Sergi for providing samples from IDDM families and $M$. Devoto and V. Brancolini for helpful statistical advice.

\section{References}

1. Tiwari J1, Terasaki PI (1985) HLA and disease associations. Springer, New York

2. Nepom GT, Erlich H (1991) MHC class II molecules and autoimmunity. Ann Rev Immunol 90: 493-525

3. Sterkers G, Zeliszewski D, Chausée AM et al. (1988) HLADQ rather than HLA-DR region might be involved in dominant non susceptibility to diabetes. Proc Natl Acad Sci USA 85: 6473-6477

4. Todd JA, Bell JI, McDevitt HO (1987) HLA-DQ $\beta$ gene contributes to susceptibility and resistance to insulin-dependent diabetes mellitus. Nature 329: 599-604

5. Morel PA, Dorman JS, Todd JA, McDevitt HO, Trucco M (1988) Aspartic acid at position 57 of the HLA-DQ $\beta$ chain protects against type I diabetes: a family study. Proc Natl Acad Sci USA 85: 8111-8115

6. Dorman JS, LaPorte RE, Stone RA, Trucco M (1990) World-wide differences in the incidence of type I diabetes are associated with amino acid variation at position 57 of the HLA-DQ $\beta$ chain. Proc Natl Acad Sci USA 87: 73707374

7. Khalil H, d'Auriol L, Gobet M et al. (1990) A combination of HLA-DQB Asp 57-negative and HLA-DQ $\alpha$ Arg 52 confers susceptibility to insulin-dependent diabetes mellitus. J Clin Invest 85: 1315-1319

8. Baish JM, Weeks T, Giles R, Hoover M, Stastny P, Capra D (1990) Analysis of HLA DQ genotypes and susceptibility in insulin-dependent diabetes mellitus. $\mathrm{N}$ Engl J Med 322: 1836-1846

9. Rønningen KS, Spurkland A, Tait BD et al. (1993) Joint report: HLA class II associations in insulin-dependent diabetes mellitus among Blacks, Caucasoids, and Japanese. 11th International Histocompatibility Workshop. In: Tsuji et al. (eds) HLA 1991. Proceedings of the 11th International Histocompatibility Workshop and Conference, vol 1. Oxford University Press, Oxford, pp 713-722

10. Kaufman JF, Auffray C, Korman AJ, Shackelford DA, Strominger JL (1984) The class II molecules of human and murine major histocompatibility complex. Cell 36: 1-13

11. Todd JH, Acha-Orbea H, Bell JI et al. (1988) A molecular basis for MHC class II-associated autoimmunity. Science 240: 1003-1009

12. Campbell RD, Trowsdale J (1993) Map of the human major histocompatibility complex. Immunol Today 14:349-352

13. Deverson EV, Gow IR, Coadwell J, Monaco JJ, Butcher GW, Howard JC (1990) MHC class II region encoding proteins related to the multidrug resistance family of transmembrane transporters. Nature 348: 738-741

14. Trowsdale J, Hanson I, Mockridge I, Beck S, Townsend A, Kelly A (1990) Sequences encoded in the class II region of the MHC related to the 'ABC' superfamily of transporters. Nature 348: 741-744

15. Spies T, Bresnahan M, Bahram S et al. (1990) A gene in the human major histocompatibility complex class II region controlling the class I antigen presentation pathway. Nature 348: 744-747
16. Morris P, Shaman J, Attaya M et al.(1994) An essential role for HLA-DM in antigen presentation by class II major histocompatibility molecules. Nature 368: 551-554

17. Fling SP, Arp B, Pious D (1994) HLA-DMA and -DMB genes are both required for MHC class II/peptide complex formation in antigen-presenting cells. Nature 368 : 554-558

18. Germain RN (1994) MHC-dependent antigen processing and peptide presentation: providing ligands for $\mathrm{T}$ lymphocyte activation. Cell 76: 287-299

19. Spies T, Cerundolo V, Colonna M, Cresswell P, Townsend A, DeMars R (1992) Presentation of viral antigen by MHC class I molecules is dependent on a putative peptide transporter heterodimer. Nature 355: 644-646

20. Neefjes JJ, Momburg F, Hämmerling GJ (1993) Selective and ATP dependent translocation of peptides by the MHC-encoded transporter. Science 261: 769-771

21. Townsend A, Öhlén C, Bastin J, Ljunggren HG, Foster L, Kärre K (1989) Association of class I major histocompatibility heavy and light chains induced by viral peptides. $\mathrm{Na}$ ture 340: $443-448$

22. Kleijmeer MI, Kelly A, Geuze HJ, Slot JW, Townsend A, Trowsdale J (1992) Location of MHC-encoded transporters in the endoplasmic reticulum and cis-Golgi. Nature 357: $342-344$

23. Powis SH, Tonks S, Mockridge I, Kelly AP, Bodmer JG, Trowsdale J (1993) Alleles and haplotypes of the MHC-encoded ABC transporters TAP1 and TAP2. Immunogenetics 37: 373-380

24. Colonna M, Bresnahan M, Bahram S, Strominger JL (1992) Allelic variants of the human putative peptide transporter involved in antigen processing. Proc Natl Acad Sci USA 89: 3932-3936

25. Rønningen KS, Undlien DE, Ploski R et al. (1993) Linkage disequilibrium between TAP2 variants and HLA class II alleles; no primary association between TAP2 variants and insulin-dependent diabetes mellitus. Eur J Immunol 23: 1050-1056

26. Caillat-Zucman S, Bertin E, Timsit J, Boitard C, Assan R, Bach JF (1993) Protection from insulin-dependent diabetes mellitus is linked to a peptide transporter gene. Eur $\mathrm{J}$ Immunol 23: 1784-1788

27. Maniatis TM, Fritsch EF, Sambrook J (1982) Molecular cloning. A laboratory manual. Cold Spring Harbor Laboratory, New York

28. Thomson G (1988) HLA disease associations: models for insulin dependent diabetes mellitus and the study of complex human genetic disorders. Annu Rev Genet 22: 31-50

29. Ott J (1991) Analysis of human genetic linkage. The Johns Hopkins University Press, Baltimore London

30. Arnheim N, Strange C, Herlich H (1985) Used of pooled DNA samples to detect linkage disequilibrium of polymorphic restriction fragments and human disease: studies of the HLA class II loci. Proc Natl Acad Sci USA 82: 69706974

31. McGinnis RE, Spielman RS (1994) Linkage disequilibrium in the insulin gene region: size variation at the $5^{\prime}$ flanking polymorphism and bimodality among "class I" alleles. Am J Hum Genet 55: 526-532

32. Martinez-Laso J, Martin-Villa JM, Alvarez M, MartinezQuiles N, Lledo G, Arnaiz-Villena A (1994) Susceptibility to insulin-dependent diabetes mellitus and short cytoplasmic ATP-binding domain TAP2*01 alleles. Tissue Antigens 44: 184-188

33. Cucca F, Congia M, Trowsdale J, Powis SH (1994) Insulindependent diabetes mellitus and the major histocompatibility complex peptide transporters TAP1 and TAP2: no as- 
sociation in a population with a high disease incidence. Tissue Antigens 44: 234-240

34. Green A, Gale EAM, Patterson CC (1992) Incidence of childhood-onset insulin-dependent diabetes mellitus: the EURODIAB ACE study. Lancet 339: 905-909

35. Jackson DJ, Capra JD (1993) TAP1 alleles in insulin-dependent diabetes mellitus: a newly defined centromeric boundary of disease susceptibility. Proc Natl Acad Sci USA 90: 11079-11083
36. Ott J (1989) Statistical properties of the haplotype relative risk. Genet Epidemiol 6: 127-130

37. Petrovsky N, Harrison LC (1995) HLA-matched control subjects are essential in studies of susceptibility to IDDM. Diabetologia 38: 125-126 (Letter)

38. Degli-Esposti MA, Andreas A, Christiansen FT, Schalke B, Albert E, Dawkins RL (1992) An approach to the localization of the susceptibility genes for generalized myasthenia gravis by mapping recombinant ancestral haplotypes. Immunogenetics 35: 355-364 